\title{
Review Paper: The Sleep Toll in Tinnitus: A Brief Review Based on the Neurofunctional Tinnitus Model
}

\author{
Iman Ghodrati Toostani $i^{1,2} \mathbb{( D}$, Mohammad Nami ${ }^{3,4,5,6^{*}} \mathbb{C}$, Tanit Ganz Sanchez ${ }^{7,8} \mathbb{D}$, Alexandre Cláudio Botazzo Delbem ${ }^{1,2} \mathbb{C}$
}

1. Neurocognitive Engineering Laboratory, Center of Engineering Applied to Health, University of São Paulo, São Carlos, Brazil.

2. Reconfigurable Computing Laboratory, Institute of Mathematics and Computer Sciences, University of São Paulo, São Carlos, Brazil.

3. Department of Neuroscience, School of Advanced Medical Sciences and Technologies, Shiraz University of Medical Sciences, Shiraz, Iran.

4. Neuroscience Laboratory-NSL (Brain, Cognition and Behavior), Department of Neuroscience, School of Advanced Medical Sciences and Technologies,

Shiraz University of Medical Sciences, Shiraz, Iran

5. Academy of Health, Senses Cultural Foundation, Sacramento, California, USA.

6. DANA Brain Health Institute, Shiraz, Iran.

7. Instituto Ganz Sanchez, São Paulo, Brazil.

8. USP-Institute of Mathematics and Computer Science, University of Sao Paulo, Sao Paulo, Brazil.

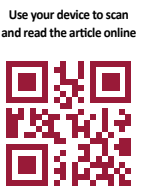

CFtation: Ghodrati Toostani I, Nami M, Sanchez TG, Delbem ACB. The Sleep Toll in Tinnitus: A Brief Review Based on the Neurofunctional Tinnitus Model. JAMSAT. 2017; 3(4):189-196. https://doi.org/10.32598/jamsat.3.4.189

doil $h$ ttps://doi.org/10.32598/jamsat.3.4.189

Article info:

Received: 12 Mar 2017

Accepted: 06 Aug 2017

Keywords:

Tinnitus, Neurofunctional tinnitus model, Sleep complaints, Insomnia, Cognitive behavior therapy

\begin{abstract}
Individuals suffering from tinnitus frequently report sleep disturbances. The most common sleep-related complaint among tinnitus patients, insomnia, may even remain unresolved despite adequate and specific treatments of tinnitus. The more severe the tinnitus, the more patients report impaired sleep. Given the fact that sleep disorders potentially affect physical and mental health, patients with tinnitus would require a special diagnostic and therapeutic care. Subjective (sleep questionnaires and self-rated psychometric evaluations) and objective (polysomnographic recording) assessments in Sleep-Disturbed Tinnitus Patients (SDTPs) have similar parameters compared to subjects with insomnia. However, as the elderly subjects have higher prevalence of organic sleep disorders, special care is needed regarding the differential diagnostic measures. Treatment of insomnia in SDTPs is commonly based on the use of hypnotics, with or without insomnia-specific psychotherapy. Similarly, cognitive behavior therapy for insomnia is shown to ameliorate both insomnia and tinnitus. This review article discusses sleep and insomnia based on a recently-proposed neurofunctional tinnitus model.
\end{abstract}

\section{Introduction}

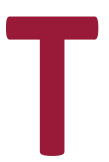

innitus is a sound perception in the absence of an external source [1]. Its prevalence has been increased considerably in epidemiological studies, so that several international scientific events and pub- lications have been devoted to explore this subject. While a recent report has shown the prevalence of tinnitus as $25.3 \%$ among US adults [2], a similar study published 15 years before reported the prevalence of tinnitus as $15 \%$ in the same population $[3,4]$. Among Brazilian children, such prevalence also reached a surprising rate of $31 \%[5]$.

* Corresponding Author:

Mohammad Nami, MD, PhD

Address: Department of Neuroscience, School of Advanced Medical Sciences and Technologies, Shiraz University of Medical Sciences, Shiraz, Iran. Tel: +98 (71) 32305471

E-mail: torabinami@sums.ac.ir 
Although many people with tinnitus do not complain about their quality of life, this symptom often causes sleeping disorders $[6,7]$, lack of concentration, impaired social life and emotional balance, contributing to the onset or worsening of anxiety and depression [8]. Nocturnal sleep problems may be described as disturbing as the daytime tinnitus by Sleep-Disturbed Tinnitus Patients (SDTPS). SDTPs may report both nocturnal and daytime problems. The special concurrence of insomnia and tinnitus is a relatively common complaint encountered when tinnitus patients refer to sleep disorder clinics or hospital-based sleep laboratories [9-11].

Sleep is in part characterized by diminished responsiveness to the external stimuli. Interestingly, some of the earliest studies trying to investigate sleep depth were designed based on the acoustic threshold. The acoustic arousal threshold seems to increase upon wake-sleep transition and during light sleep and decrease in deep sleep [12]. As such, any undesired noise level above this threshold tends to interfere with sleep. Over the past few years, a considerable number of patients with tinnitus referring to our clinics and hospital-based sleep laboratory have complained of hard-to-tackle insomnia. Being an internally perceived acoustic stimulus, tinnitus has attracted special interest in various aspects of sleep research, mainly insomnia. However, the available literature on insomnia and tinnitus seems to be scant and mixed $[7,13,14]$.

Recently, Toostani et al. proposed a Neurofunctional Tinnitus Model (NfTM) to structurally illustrate the tinnitus network through the peripheral auditory system; auditory cortex; brainstem (raphe nucleus); thalamus (reticular, medial geniculate, and dorsal nuclei); limbic system (anterior cingulate cortex, amygdala); subcallosal; and paralimbic areas including basal ganglia (ventral palladium), striatum (nucleus accumbens) and ventromedial prefrontal cortex. Over developing procedure of clinical distress stage of NFTM, the general suspicion or disease-indication of patients can turn the neutral phantom sound related cognitive-emotional interpretation into negative one that can emerge the reactions via sympathetic nervous system. This negative appraisal and evaluative conditioned learning mechanism enhance the associated cognitive-emotional value and contribute to the persistence of the perceived sound $[15,16]$.

Taking the proposed NfTM into accounts, some questions need to be addressed: 1 . To what extent the sleep disturbance experienced by tinnitus patients is perceived as a direct consequence of tinnitus or a predisposing factor for tinnitus to appear and reinforce the previous sleep disturbance?; 2 . If such an association is logical and linear, why only some patients with tinnitus report insomnia?; and 3. When tinnitus is seen as a persistent sleep-disturbing stimulus, what could be the most efficient therapeutic options based on the NfTM model?

\section{Epidemiological insights on tinnitus and insomnia}

SDTPs usually blame tinnitus for their insomnia. Studies have proposed sleep problems among the most prevalent tinnitus complaints, together with hearing loss, emotional stress and anxiety [17-21]. Several investigations have reported varied prevalence of sleep disturbances among tinnitus subjects ranging from $30 \%$ to $80 \%[9,11,13,22,23]$. For example, one report has demonstrated that up to $60 \%$ of tinnitus sufferers experience initial insomnia [24], while a larger-sample study has contradictorily shown that nearly $15 \%$ of tinnitus patients report sleep difficulties [25].

The clear disparity in such epidemiological reports may largely depend on whether the sample comprises tinnitus sufferers [25], cases with tinnitus [13], general population [7, 17, 19, 22], or a specific subpopulation group [18, 21, 26]. Moreover, the sleep quality could vary based on the investigation time. Other contributing factors might include gender, age, medication history, psychological and neurocognitive profile as well as subjects' social history and sleep hygiene status [7].

Patients with recent onset tinnitus are generally more susceptible to insomnia [13]. In fact, nearly half of the subjects who experience tinnitus for less than 1 year tend to report sleep complaints, whereas only one fourth of the individuals with long-lasting tinnitus (10 years and more) are likely to report the same [14]. On the other hand, patients with tinnitus appear to demonstrate both acute and chronic types of insomnia. Nevertheless, whether such insomnia patterns represent distinct types or merely a variation form still needs to be elucidated. Chronic insomnia with coexisting tinnitus becomes even more debilitating since the patients suffer from both conditions for a longer duration. Followup studies in SDTPs have indicated that almost $25 \%$ of adequately-treated tinnitus patients may continue to have long-term sleep complaints namely insomnia.

In long-term follow-up (over 5 years), more than $60 \%$ of patients with tinnitus keep on reporting sleep problems despite treatment [27], Moreover, SDTPs generally report louder and more intense subjective sense of tinnitus $[7,11,28]$. These reports show, but do not explain, why sleep problems - mainly initial insomnia - persist 
in some subjects with tinnitus. The issue which has become a difficult clinical challenge both upon diagnosis and treatment.

\section{The clinical data of sleep-disturbed tinnitus patients}

SDTPs tend to report initial insomnia due to tinnitus rather than tinnitus-induced Wakes After Sleep Onset (WASO). However, both types of sleep disturbances might coexist in up to $40 \%$ of tinnitus sufferers $[9,10]$. There are contradictory reports on the association between the tinnitus loudness and sleep disturbance. The NfTM projected two different scenarios in different stages of tinnitus development. During neutral stage contingency of perceiving phantom sound and difficulty in falling sleep might be accompanied by dislike feeling of inability to sleep and stress and results in strengthening the negative cognitive-emotional value of sound by means of evaluative conditioned learning mechanism, leading to the comorbidity of tinnitus in insomnia patients.

In the second scenario, over the NfTM clinical distress stage, perceiving tinnitus in the absence of daily attentional inputs can result in reinforcement of dislike feeling which stimulates autonomic discharges (including tachycardia, perspiration, etc.) potentially leading to chronic insomnia $[15,16]$. Considering the NfTM, insomnia facilitates turning tinnitus into a clinical distress while it does not generate tinnitus. The hypothesis that tinnitus can be a cause for insomnia has been tested and confirmed in several studies [29-35].

While some reports demonstrated a linear relationship between tinnitus loudness and sleep insufficiency [19], others failed to prove the same relationship [10, 36]. In fact, evidence suggests that only some tinnitus patients report their sleep problems with tinnitus [7]. In other words, despite the fact that SDTPs are predominantly have prolonged sleep onset latency, the majority of tinnitus sufferers report sleep insufficiency regardless of tinnitus problem. Given the fact that subjective sleep assessments are based on subjects' memory, such studies may only offer limited dependable data on true influence of tinnitus on sleep. From the subjective perspective, insomniacs usually underestimate their sleep [7, 22]. There exist few published reports assessing the tinnitus sufferers' sleep using the full-polysomnographic recording setup [10].

An objective evaluation has suggested that Total Sleep Time (TST) of insomniacs with no tinnitus is significantly shorter than healthy controls [17]. However, comparing subjective and objective sleep parameters, together with clinical data on insomnia, daytime somnolence, fatigue and mood changes revealed no differences between primary insomniacs and SDTPs based on their objective sleep measures [7], subjective scores on Excessive Daytime Sleepiness (EDS) and mood changes. Meanwhile, impaired sleep efficiency and the prolonged sleep onset latency was reported as a common finding in both groups [7]. With respect to cognitive performance and sustained attention in particular, studies have yielded comparable results in sleep deprived subjects with and without tinnitus.

Findings from well-designed studies have proposed that sleep insufficiency in tinnitus patients is partially linked to their clinical distress symptoms [19, 33]. Patients whose sleep was most disturbed scored significantly greater on their tinnitus annoyance in the evening. Meanwhile, measures of mood and emotional distress did not relate to the degree of sleep disturbance. In addition, polysomnographic studies have shown that objective sleep parameters are similar in SDTPs and insomniacs [10, 12, 19]. Assuming the Tinnitus Handicap Inventory (THI) as an outcome, no correlation was found between its increased score with decreased time of REM in the tinnitus group ( $r=0.04)$. However, a mild correlation was found with the increase of light sleep time (stages 1 and 2). Therefore, patients with prolonged light sleep report a higher annoyance by THI score $(r=0.4)$ [19].

\section{Adjustment and psychophysiological insomnia}

The fact that only some patients with tinnitus develop insomnia may be a consequence of sleep disturbance and tinnitus following different somatic covariates. Hence, the question whether insomnia in patients with tinnitus is a consequence of tinnitus or just comorbidity still remains open. Perhaps a fraction of patients with acute tinnitus suffer from adjustment insomnia, where the presence of a clear stressor is a key contributor and sleep complaints significantly improve once the stressor is removed. The clinical observation that the duration of tinnitus has an inverse correlation with the presence of insomnia could be justified by spontaneous tinnitus habituation in SDTPs [37].

On the other hand, when insomnia persists in patients with chronic tinnitus, the condition does not recount the criteria of adjustment insomnia. In some instances, although tinnitus is seen largely responsible for disturbed sleep both by the patients and physicians, no clear precipitating factor is identified. Then, one plausible condition to consider is the psychophysiological insomnia [38, 39]. In such cases, patients are preconditioned with low threshold in somatic and mental arousal where symp- 
toms revolve around the urge for sleep, awareness on inability to sleep and sleep-related cues [38].

As such, several aspects of tinnitus psychology may overlap with the characteristics of psychophysiological insomnia. Classical conditioning, selective attention and tinnitus appraisal are among the measures used to ameliorate tinnitus symptoms [40]. Meanwhile, similar concepts are applied in the Cognitive Behavioral Therapy (CBT) of psychophysiological insomnia [41]. Except for the classical conditioning part, all remaining features of the above are in agreement with the NfTM $[15,16]$.

Emerging evidence together with NfTM $[15,16]$ support the notion that clinical distress stage of tinnitus and insomnia reinforce each other [26]. In acute tinnitus, many sufferers perceive the condition as sleep-preventing. Being perceived as sustainable triggers, tinnitus, obsession on sleep, selective attention toward the problem, difficulty in falling asleep and mental/somatic hyperarousal may contribute to a debilitating vicious cycle [7]. In an interim analysis of our clinical experience in a sleep disorders laboratory, a clear correlation is being noted between the severity of tinnitus and the extent of sleep disturbance (unpublished data).

Medical, neurocognitive, and social consequences of chronic insomnia

Chronic insomnia is deemed as a true health issue and inefficient sleep. As a direct consequence, Excessive Daytime Sleepiness (EDS) and impaired diurnal functioning are common symptoms and help defining the criteria of insomnia [42]. A considerable body of evidence has substantiated that insomnia potentially influences physical and mental health [43], hence needs to be addressed as a potential medical problem. Other secondary risks of chronic insomnia include arterial hypertension [44], ischemic heart diseases, depression, impaired professional productiveness [45], and motor vehicle accidents [46]. It has been documented that nearly $25 \%-30 \%$ of automobile collisions are potentially associated with driving fatigue. Drowsy driving is not only a personal but also a public hazard and commercial drivers with alternate shifts are critically at risk [47].

Furthermore, the appropriate treatment of insomnia is shown to improve patients' daytime vigilance and neurocognitive agility parameters such as attention, memory, and executive function represented by reduced reaction time upon driving. Current sleep research findings indicate that many people with untreated sleep disorders such as insomnia still involve in high-risk jobs including public transporta- tion [46]. Therefore, in SDTPs, drowsy-driving issue, and the underlying medical factors should be taken into consideration. Well-designed studies seem to be needed to address the prevalence of this syndrome among the population and the public transport drivers in particular.

\section{Therapy}

Insomnia is treated through pharmacological and nonpharmacological (cognitive behavior therapy for insomnia or (BT-I) approaches [48, 49]. Benzodiazepines and sedative antidepressants are shown to retain not only limited benefits but also potential drawbacks in longterm use [50-54]. This holds true for $\gamma$-aminobutyric acid (GABA)-A receptor agonists (Z-class medications) including zolpidem, zopiclone, and zaleplon [49]. Besides the issue of tolerance, the chronic use of hypnotics is subject to precautions [53].

However, some reports have demonstrated that 6 months use of Z-class hypnotics may be well-tolerated with no potential harm [55]. Either way, given the fact that SDTPs commonly require treatments for an even longer duration, it seems that in many instances, CBT-I is the preferred approach to target the symptoms and modulate distress $[56,57]$. Evidence has supported the fact that CBT-I components, including stimulus control, bed time restriction, meditative therapy, and sleep hygiene can provide positive and sustainable effects on primary insomnia $[49,58]$. Studies have substantiated that neurotransmitters including glutamate, GABA, serotonin, dopamine and Acetylcholine (ACh) are contributing in tinnitus generation.

The crucial role of serotonin (5-HT) was established in sleep-wake cycles $[59,60]$ and its deficit causes insomnia. Considering the anatomy of central auditory pathways, the distribution of neurotransmitters may be as follows: serotonergic neurons of brainstem are mainly originated from Dorsal Raphe Nucleus (DRN) [61-63]. Thalamus reticular nucleus and dorsal thalamus trigger by serotonergic neurons which receive projects from DRN, Nucleus Accumbens (NAc) and paralimbic area $[64,65]$. GABAergic neurons of TRN stimulate by serotonin $[66,67]$ that results in inhibiting of thalamic relay in sensory sectors [68]. The TRN inhibition may shift between tonic and burst firing mode of thalamocortical relay $[69,70]$. Recently, DRN was also proposed as taking part of tinnitus connectivity network within the NfTM $[15,16]$.

Psychologically-stressed subjects are shown to have lower levels of serotonin concentration in their blood 
samples, hence the use of serotonin reuptake inhibitors is expected to ameliorate symptoms in some patients [71]. Assessment of the blood serotonin level in tinnitus sufferers versus controls revealed that patients with tinnitus have significantly higher levels of serotonin concentration [72]. Similarly, some preclinical studies have found that increased serotonin levels induced by salicylate may be attributed to changes in neuronal activity and tinnitus [73]. Clinical experience has also confirmed that the use of Selective Serotonin Reuptake Inhibitors (SSRI) may provoke or aggravate tinnitus rather than ameliorating it [74]. The association between stress and tinnitus and the possible role of serotonin in such association need further explanation.

Apart from the pharmacological approach, technological advances in sound-based and cortical stimulation have contributed to tinnitus treatment. Sound stimulation during sleep is a new strategy towards the treatment of idiopathic subjective tinnitus. This treatment is based on the notion that the auditory system keeps working during sleep by processing the incoming information. Empirical evidence with such therapy has substantiated that tinnitus intensity continues to decrease within weeks, with some SDTPs experiencing periods of complete silence [23]. This method of treatment is against the NfTM fundamental assumption in which conscious perception and attention play an important role in tinnitus development $[15,16]$.

Tinnitus retraining therapy and CBT-I have demonstrated acceptable and comparable efficacy in the treatment of subjective tinnitus. However, further research using objective measurements (i.e. polysomnography) would help to evaluate the effect of both approaches in SDTPs [75]. Additionally, remediating the unfavorable behavioral patterns and dysfunctional attitudes are expected to help SDTPs regaining pre-sleep ease when psychological insomnia is suspected [76].

With regard to the assessment of results following the treatments, the Pittsburgher Sleep Quality Index (PSQI) is among the most preferred validated sleep questionnaires used in the practice of sleep medicine [77]. This questionnaire is a self-rated 19-item tool used to assess the subjects' sleep quality over the past month. Its subscales comprise sleep duration (PSQIDURAT), sleep latency (PSQILATEN), sleep disturbances (PSQIDISTB), subjective sleep quality (PSQIQUAL), use of sleeping medication (PSQIMEDS) and day-time dysfunction (PSQIDAYDYS) [78].

Items are scored from 0 (without difficulty) to 3 (maximal difficulty). The total scores of all components yields the global PSQI score (ranging from 0 to 21). The higher the total PSQI score, the sleep quality will be poorer. This subjective assessment tool is recommended to evaluate the efficacy and safety of the treatments for the management of sleep disturbances in patients with tinnitus, too [79].

Other sleep disturbances in patients with tinnitus Sleep Apnea Syndrome (SAS) (obstructive or central type) and Periodic Leg Movements during Sleep (PLMS) may be concurrently observed in up to $40 \%$ of SDTPs [80]. Diagnosing primary sleep disorders in SDTPs becomes particularly important since mild sleep insufficiencies due to SAS or PLMS might result in sleep fragmentation and synergize the sleep disturbance caused by insomnia in SDTPs. Such patients generally report difficulty falling back asleep upon WASO.

To our knowledge, there is no report on the prevalence of sleep-related breathing disorders in patients with tinnitus, nor on the impact of Continuous Positive Airway Pressure (CPAP) therapy on the severity of sleep disturbance in tinnitus sufferers with SAS. The fact that benzodiazepines and other sedative agents which are used to control tinnitus symptoms may aggravate sleep-related breathing disorders should be considered in treating the patients [81].

In addition, PLMS may also exert further impairing effects in the sleep efficiency of SDTPs. The condition is characterized by stereotyped and repetitive leg movements during sleep. Studies have proposed that PLMS occurs in up to $20 \%$ of insomniacs and its prevalence increases by age $[17,19,79]$. Likewise, some sedative drugs used to treat insomnia may worsen PLMS symptoms [22].

\section{Conclusion}

Insomnia and tinnitus which may coexist or contribute to each other are chronically-debilitating conditions affecting the quality of life. When sleep disturbances (particularly insomnia) are being addressed in tinnitus sufferers, it is essential to consider organic sleep disorders. The NfTM may be used to develop specific treatment measures to help SDTPs.

\section{Ethical Considerations}

\section{Funding}

This research was financially supported by the Coordenação de Aperfeiçoamento de Pessoal de Nível Superior (CAPES) and the Center for Research, Inno- 
vation and Diffusion of Mathematical Sciences Center Applied to Industry (CEPID-CeMEAI) of Sao Paulo Research Foundation (FAPESP), affiliated to the Institute of Mathematics and Computer Sciences (ICMC) USP São Carlos.

\section{Conflict of interest}

Authors declared no conflict of interest.

\section{References}

[1] Mc Fadden D. Tinnitus: Facts, theories and treatments. Washington, D.C.: National Academy Press; 1982.

[2] Shargorodsky J, Curhan GC, Farwell WR. Prevalence and characteristics of tinnitus among US adults. The American Journal of Medicine. 2010; 23(8):711-8. [DOI:10.1016/j.amjmed.2010.02.015] [PMID]

[3] Disorders NloDaOC. National strategic Research Plan. Smell, taste and touch and chemosensory disorders. Bethesda: US Department of Health and Human Services, Public Health Service, National Institutes of Health; 1995. No. 95-3711.

[4] Hoffman HJ, Reed GW. Epidemiology of tinnitus. In: Snow JB (Ed), Tinnitus: Theory and management. Ontario: Hamilton; 2004.

[5] Coelho CB, Sanchez TG, Tyler RS. Tinnitus in children and associated risk factors. Progress in Brain Research. 2007; 166:179-91. [DOI:10.1016/S0079-6123(07)66016-6]

[6] Coelho CC, Sanchez TG, Bento RF. [Characteristics of tinnitus in patients attended at referral service (Portuguese)]. International Archives of Otorhinolaryngology. 2004; 8(3):216-24.

[7] Cronlein T, Langguth B, Geisler P, Hajak G. Tinnitus and insomnia. Progress in Brain Research. 2007; 166:227-33. [DOI:10.1016/S00796123(07)66021-X]

[8] Langguth B, Kleinjung T, Fischer B, Hajak G, Eichhammer P, Sand PG. Tinnitus severity, depression, and the big five personality traits. Progress in Brain Research. 2007; 166:221-5. [DOI:10.1016/S00796123(07)66020-8]

[9] Hebert S, Carrier J. Sleep complaints in elderly tinnitus patients: A controlled study. Ear and Hearing. 2007; 28(5):649-55. [DOI:10.1097/ AUD.0b013e31812f71cc] [PMID]

[10] Hebert S, Fullum S, Carrier J. Polysomnographic and quantitative electroencephalographic correlates of subjective sleep complaints in chronic tinnitus. Journal of Sleep Research. 2011; 20(1 Pt 1):38-44. [DOI:10.1111/j.1365-2869.2010.00860.x] [PMID]

[11] Izuhara K, Wada K, Nakamura K, Tamai Y, Tsuji M, Ito Y, et al. Association between tinnitus and sleep disorders in the general Japanese population. Annals of Otology, Rhinology, and Laryngology. 2013; 122(11):701-6. [DOI:10.1177/000348941312201107] [PMID]

[12] Wallhausser-Franke E, Schredl M, Delb W. Tinnitus and insomnia: Is hyperarousal the common denominator? Sleep Medicine Reviews. 2013; 17(1):65-74. [DOI:10.1016/j.smrv.2012.04.003] [PMID]

[13] Folmer RL, Griest SE. Tinnitus and insomnia. American Journal of Otolaryngology. 2000; 21(5):287-93. [DOI:10.1053/ajot.2000.9871] [PMID]
[14] Miguel GS, Yaremchuk K, Roth T, Peterson E. The effect of insomnia on tinnitus. Annals of Otology, Rhinology, and Laryngology. 2014 123(10):696-700. [DOI:10.1177/0003489414532779] [PMID]

[15] Ghodratitoostani I, Zana Y, Delbem ACB, Sani SS, Ekhtiari H, Sanchez TG. Theoretical tinnitus framework: A neurofunctional model. Frontiers in Neuroscience. 2015; 10:370. [DOI:10.3389/fnins.2016.00370] [PMID] [PMCID]

[16] Godrati Toostani I. Tinnitus: A nneuro-functionational Tinntius Model [PhD thesis]. Santo André: University Federal ABC; 2013.

[17] Alster J, Shemesh Z, Ornan M, Attias J. Sleep disturbance associated with chronic tinnitus. Biological Psychiatry. 1993; 34(1-2):84-90. [DOI:10.1016/0006-3223(93)90260-K]

[18] Asplund R. Sleepiness and sleep in elderly persons with tinnitus. Archives of Gerontology and Geriatrics. 2003; 37(2):139-45. [DOI:10.1016/S0167-4943(03)00028-1]

[19] Attanasio G, Russo FY, Roukos R, Covelli E, Cartocci G, Saponara $M$. Sleep architecture variation in chronic tinnitus patients. Ear and Hearing. 2013; 34(4):503-7. [DOI:10.1097/AUD.0b013e31827bc436] [PMID]

[20] Eysel Gosepath K, Selivanova O. [Characterization of sleep disturbance in patients with tinnitus (German)]. Laryngo-Rhino-Otologie. 2005; 84(5):323-7. [DOI:10.1055/s-2005-861020] [PMID]

[21] Fernandes G, Siqueira JT, Godoi Goncalves DA, Camparis CM. Association between painful temporomandibular disorders, sleep bruxism and tinnitus. Brazilian Oral Research. 2014; 28(1):1-7. [DOI:10.1590/1807-3107BOR-2014.vol28.0003]

[22] Fioretti AB, Fusetti M, Eibenstein A. Association between sleep disorders, hyperacusis and tinnitus: Evaluation with tinnitus questionnaires. Noise \& Health. 2013; 15(63):91-5. [DOI:10.4103/14631741.110287] [PMID]

[23] Pedemonte M, Drexler D, Rodio S, Geisinger D, Bianco A, Pol-Fernandes $D$, et al. Tinnitus treatment with sound stimulation during sleep. International Tinnitus Journal. 2010; 16(1):37-43. [PMID]

[24] Tyler RS, Baker L. Difficulties experienced by tinnitus sufferers. The Journal of Speech and Hearing Disorders. 1983; 48(2):150-4 [DOI:10.1044/jshd.4802.150]

[25] Sanchez L, Stephens D. A tinnitus problem questionnaire in a clinic population. Ear and Hearing. 1997; 18(3):210-7. [DOI:10.1097/00003446-199706000-00004] [PMID]

[26] Zheng Y, Stiles L, Chien YT, Darlington CL, Smith PF. The effects of acute stress-induced sleep disturbance on acoustic trauma-induced tinnitus in rats. BioMed Research International. 2014; 2014:724195. [DOI:10.1155/2014/724195] [PMID] [PMCID]

[27] Andersson G, Vretblad P, Larsen HC, Lyttkens L. Longitudinal followup of tinnitus complaints. Archives of Otolaryngology--Head \& Neck Surgery. 2001; 127(2):175-9. [DOI:10.1001/archotol.127.2.175]

[28] Lasisi AO, Gureje O. Prevalence of insomnia and impact on quality of life among community elderly subjects with tinnitus. Annals of Otology, Rhinology, and Laryngology. 2011; 120(4):226-30. [DOI:10.1 177/000348941112000402] [PMID] [PMCID]

[29] Wallhäusser-Franke E, Schredl M, Delb W. Tinnitus and insomnia Is hyperarousal the common denominator? Sleep Medicine Reviews. 2013; 17(1):65-74. [DOI:10.1016/j.smrv.2012.04.003] [PMID] 
[30] Miguel GS, Yaremchuk K, Roth T, Peterson E. The effect of insomnia on tinnitus. Annals of Otology, Rhinology \& Laryngology. 2014; 123(10):696-700. [DOI:10.1177/0003489414532779] [PMID]

[31] Kreuzer PM, Vielsmeier V, Langguth B. Chronic tinnitus: An interdisciplinary challenge. Archive of "Deutsches Ärzteblatt International. 2013; 110(16):278. [DOI:10.3238/arztebl.2013.0278]

[32] Hebert S, Fullum S, Carrier J. Polysomnographic and quantitative electroencephalographic correlates of subjective sleep complaints in chronic tinnitus. Journal of Sleep Research. 2011; 20(1pt1):38-44. [DOI:10.1111/j.1365-2869.2010.00860.x] [PMID]

[33] Hallam R. Correlates of sleep disturbance in chronic distressing tinnitus. Scandinavian Audiology. 1996; 25(4):263-6. [DOI:10.3109/01050399609074965] [PMID]

[34] Alster J, Shemesh Z, Ornan M, Attias J. Sleep disturbance associated with chronic tinnitus. Biological Psychiatry. 1993; 34(1):84-90. [DOI:10.1016/0006-3223(93)90260-K]

[35] Joo YH, Han KD, Park KH. Association of hearing loss and tinnitus with health-related quality of life: The korea national health and nutrition examination survey. PloS One. 2015; 10(6):e0131247. [DOI:10.1371/journal.pone.0131247] [PMID] [PMCID]

[36] Moon P. Emotional distress but not tinnitus loudness affects quality of sleep in tinnitus patients [PhD thesis]. São Paulo: University of São Paulo; 2015.

[37] Jansson-Frojmark $M$. The work and social adjustment scale as a measure of dysfunction in chronic insomnia: Reliability and validity. Behavioural and Cognitive Pychotherapy. 2014; 42(2):186-98. [DOI:10.1017/S135246581200104X] [PMID]

[38] Daniel L, Kazak AE, Li Y, Hobbie W, Ginsberg J, Butler E, et al. Relationship between sleep problems and psychological outcomes in adolescent and young adult cancer survivors and controls. Supportive Care in Cancer. 2016; 24(2):539-46. [DOI:10.1007/s00520-015-2798-2] [PMID] [PMCID]

[39] Segura-Jimenez V, Carbonell-Baeza A, Keating XD, Ruiz JR, CastroPinero J. Association of sleep patterns with psychological positive health and health complaints in children and adolescents. Quality of Life Research. 2015; 24(4):885-95. [DOI:10.1007/s11136-014-0827-0] [PMID]

[40] Andersson G, Westin V. Understanding tinnitus distress: Introducing the concepts of moderators and mediators. International Journal of Audiology. 2008; 47(2):S106-11. [DOI:10.1080/14992020802301670] [PMID]

[41] Espie CA, Broomfield NM, MacMahon KM, Macphee LM, Taylor LM. The attention-intention-effort pathway in the development of psychophysiologic insomnia: A theoretical review. Sleep Medicine Reviews. 2006; 10(4):215-45. [DOI:10.1016/j.smrv.2006.03.002] [PMID]

[42] Chung KF, Yeung WF, Ho FY, Yung KP, Yu YM, Kwok CW. Cross-cultural and comparative epidemiology of insomnia: The Diagnostic and Statistical Manual (DSM), International Classification of Diseases (ICD) and International Classification of Sleep Disorders (ICSD). Sleep Medicine. 2015; 16(4):477-82. [DOI:10.1016/j.sleep.2014.10.018] [PMID]

[43] Riemann D, Voderholzer U. Primary insomnia: A risk factor to develop depression. Journal of Affective Disorders. 2003; 76(1-3):255-9. [DOI:10.1016/S0165-0327(02)00072-1]

[44] Li Y, Vgontzas AN, Fernandez-Mendoza J, Bixler EO, Sun Y, Zhou J, et al. Insomnia with physiological hyperarousal is associated with hypertension. Hypertension. 2015; 65(3):644-50. [DOI:10.1161/HYPERTENSIONAHA.114.04604] [PMID]
[45] Hall MH, Kline CE, Nowakowski S. Insomnia and sleep apnea in midlife women: Prevalence and consequences to health and functioning. F1000prime Reports. 2015; 7:63. [DOI:10.12703/P7-63] [PMID] [PMCID]

[46] Torabi-Nami M, Mehrabi S. [Drowsy driving and road accidents; screening and treating the sleep-related underlying causes (Persian)]. Paper presented at The $5^{\text {th }}$ International Conference on Reducing Burden of Traffic Accidents; Challenges and Strategies. 13 September 2014; Shiraz, Iran.

[47] Ebrahimi MH, Sadeghi M, Dehghani M, Niiat KS. Sleep habits and road traffic accident risk for Iranian occupational drivers. International Journal of Occupational Medicine and Environmental Health. 2015 28(2):305-12. [DOI:10.13075/ijomeh.1896.00360]

[48] Morin CM, Bootzin RR, Buysse DJ, Edinger JD, Espie CA, Lichstein KL. Psychological and behavioral treatment of insomnia:update of the recent evidence (1998-2004). Sleep. 2006; 29(11):1398-414. [DOI:10.1093/sleep/29.11.1398] [PMID]

[49] Torabi-Nami M. Chronic insomnia, pharmacotherapy and the cognitive behavioral approaches. Sleep Disorders \& Therapy. 2014; 3(1):1000151. [DOI:10.4172/2167-0277.1000151]

[50] van Dam JG. [Sleeping poorly due to sedatives (rebound insomnia) and the role of zopiclone (Dutch)]. Dutch Journal of Medicine. 1992; 136(24):1175-6. [PMID]

[51] McCree DH. The appropriate use of sedatives and hypnotics in geriatric insomnia. American Pharmacy. 1989; 29(5):49-55. [DOI:10.1016/ S0160-3450(15)31787-6]

[52] Taylor HL, Rybarczyk BD, Nay W, Leszczyszyn D. Effectiveness of a CBT intervention for persistent insomnia and hypnotic dependency in an outpatient psychiatry clinic. Journal of Clinical Psychology. 2015; 71(7):666-83. [DOI:10.1002/jclp.22186] [PMID]

[53] Chung KH, Li CY, Kuo SY, Sithole T, Liu WW, Chung MH. Risk of psychiatric disorders in patients with chronic insomnia and sedativehypnotic prescription: A nationwide population-based follow-up study. Journal of Clinical Sleep. 2015; 11(5):543-51. [DOI:10.5664/ jcsm.4700]

[54] Scalo J, Desai P, Rascati K. Insomnia, hypnotic use, and health-related quality of life in a nationally representative sample. Quality of Life Research. 2015; 24(5):1223-33. [DOI:10.1007/s11136-014-08421] [PMID]

[55] Krystal AD, Walsh JK, Laska E, Caron J, Amato DA, Wessel TC, et al. Sustained efficacy of eszopiclone over 6 months of nightly treatment: Results of a randomized, double-blind, placebo-controlled study in adults with chronic insomnia. Sleep. 2003; 26(7):793-9. [DOI:10.1093/ sleep/26.7.793] [PMID]

[56] Beukes EW, Manchaiah V, Allen PM, Baguley DM, Andersson G. Internet-based cognitive behavioural therapy for adults with tinnitus in the UK: Study protocol for a randomised controlled trial. BMJ Open. 2015; 5(9):e008241. [DOI:10.1136/bmjopen-2015-008241] [PMID] [PMCID]

[57] Conrad I, Kleinstauber M, Jasper K, Hiller W, Andersson G, Weise C. The changeability and predictive value of dysfunctional cognitions in cognitive behavior therapy for chronic tinnitus. International Journal of Behavioral Medicine. 2015; 22(2):239-50. [DOI:10.1007/s12529 014-9425-3] [PMID]

[58] Torabi-Nami M. "Sleep hygiene" The gateway for efficient sleep: A brief review. WMC Brain. 2011; 2(9):WMC002245. 
[59] Pace-Schott E, Hobson J. Basic mechanisms of sleep: New evidence on the neuroanatomy and neuromodulation of the NREM-REM cycle. In: Pace-Schott E, Hobson J, (Eds). American College of Neuropsychopharmacology: The fifth generation of progress. Philadelphia: Lippincott, Williams and Wilkins; 2002.

[60] Monti JM. Primary and secondary insomnia: Prevalence, causes and current therapeutics. Current Medicinal Chemistry-Central Nervous System Agents. 2004; 4(2):119-37. [DOI:10.2174/1568015043357039]

[61] Gallager DW, Aghajanian GK. Effect of antipsychotic drugs on the firing of dorsal raphe cells. II. Reversal by picrotoxin. European Journal of Pharmacology. 1976; 39(2):357-64. [DOI:10.1016/00142999(76)90145-X]

[62] Gallager DW, Aghajanian GK. Effect of antipsychotic drugs on the firing of dorsal raphe cells. I. Role of adrenergic system. European Journal of Pharmacology. 1976; 39(2):341-55. [DOI:10.1016/00142999(76)90144-8]

[63] Wang RY, Gallager DW, Aghajanian GK. Stimulation of pontine reticular formation suppresses firing of serotonergic neuronses in the dorsal raphe. Nature. 1976; 264(5584):365-8. [DOI:10.1038/264365a0] [PMID]

[64] Brown P, Molliver ME. Dual serotonin (5-HT) projections to the nucleus accumbens core and shell: Relation of the 5-HT transporter to amphetamine-induced neurotoxicity. The Journal of Neuroscience. 2000; 20(5):1952-63. [DOI:10.1523/JNEUROSCI.20-05-01952.2000] [PMID]

[65] O'Donnell P, Lavin A, Enquist LW, Grace AA, Card JP. Interconnected parallel circuits between rat nucleus accumbens and thalamus revealed by retrograde transynaptic transport of pseudorabies virus. The Journal of Neuroscience. 1997; 17(6):2143-67. [DOI:10.1523/ JNEUROSCI.17-06-02143.1997] [PMID]

[66] McCormick DA, Wang Z. Serotonin and noradrenaline excite GABAergic neurones of the guinea-pig and cat nucleus reticularis thalami. The Journal of Physiology. 1991; 442:235-55. [DOI:10.1113/ jphysiol.1991.sp018791]

[67] Pape HC, McCormick DA. Noradrenaline and serotonin selectively modulate thalamic burst firing by enhancing a hyperpolarization-activated cation current. Nature. 1989; 340(6236):715-8. [DOI:10.1038/340715a0] [PMID]

[68] Guillery RW, Sherman SM. Thalamic relay functions and their role in corticocortical communication: Generalizations from the visual system. Neuron. 2002; 33(2):163-75. [DOI:10.1016/S08966273(01)00582-7]

[69] Llinas $R$, Jahnsen $H$. Electrophysiology of mammalian thalamic neurones in vitro. Nature. 1982; 297(5865):406-8. [DOI:10.1038/297406a0] [PMID]

[70] Sherman SM. A wake-up call from the thalamus. Nature Neuroscience. 2001; 4(4):344-6. [DOI:10.1038/85973] [PMID]

[71] Ishiwata. H, Shiga. T, Okado. N. Selective serotonin reuptake inhibitor treatment of early postnatal mice reverses their prenatal stressinduced brain dysfunction. Neuroscience. 2005; 133(4):893-901. [DOI:10.1016/j.neuroscience.2005.03.048] [PMID]

[72] Sachanska. T. Changes in blood serotonin in patients with tinnitus and other vestibular disturbances. The International Tinnitus Journal. 1999; 5(1):24-6. [PMID]

[73] Wei S, Jianzhong L, Erin L. Neurotransmitter modulation relates with tinnitus signal generation and management. Journal of Otology. 2007; 2(2):63-9. [DOI:10.1016/S1672-2930(07)50016-4]
[74] Robinson SK, Viirre ES, Stein MB. Antidepressant therapy in tin nitus. Hearing Research. 2007; 226(1-2):221-31. [DOI:10.1016/j heares.2006.08.004] [PMID]

[75] Martinez-Devesa P, Perera R, Theodoulou M, Waddell A. Cognitive behavioural therapy for tinnitus. Cochrane Database of Systematic Reviews. 2010; 2010(9):CD005233. [DOI:10.1002/14651858. CD005233.pub3]

[76] Cima RF, Andersson G, Schmidt CJ, Henry JA. Cognitive-behaviora treatments for tinnitus: A review of the literature. Journal of the American Academy of Audiology. 2014; 25(1):29-61. [DOI:10.3766/ jaaa.25.1.4] [PMID]

[77] Torabi-Nami M, Saremi AA, Madadi G. Quantification of subjective sleep quality in healthy nulliparous pregnant, post partum and post menopausal women in Shiraz, using the pittsburg sleep quality index. The Internet Journal of Gynecology and Obstetrics. 2011; 15(1):1.

[78] Espie CA, Kyle SD, Hames P, Gardani M, Fleming L, Cape J. The sleep condition indicator: A clinical screening tool to evaluate insomnia disorder. BMJ Open. 2014; 4:e004183. [DOI:10.1136/bmjopen-2013-004183] [PMID] [PMCID]

[79] Burgos I, Feige B, Hornyak B, Härter M, Weske-Heck S, Voderholzer $R$, et al. Chronic tinnitus and associated sleep disturbances. Somnologie. 2005; 9(3):133-8. [DOI:10.1111/j.1439-054X.2005.00056.x]

[80] Fioretti AB, Fusetti M, Eibenstein A. Association between sleep disorders, hyperacusis and tinnitus: Evaluation with tinnitus questionnaires. Noise \& Health. 2013; 15(63):91-5. [DOI:10.4103/14631741.110287] [PMID]

[81] Smith TE. Tinnitus and Sleep Apnea. Neurology Now. 2012 8(2):4-8. [DOI:10.1097/01.NNN.0000414200.16892.78] 